\title{
TWO FAMILIES OF SYMMETRY-PRESERVING REVERSIBLE INTEGER-TO-INTEGER WAVELET TRANSFORMS
}

\author{
Michael D. Adams and Rabab Ward \\ Dept. of Elec. and Comp. Eng., University of British Columbia, 2356 Main Mall, Vancouver, BC, Canada V6T 1Z4 \\ mdadams@ieee.org and rababw@ece.ubc.ca
}

\begin{abstract}
Two families of symmetry-preserving reversible integer-to-integer wavelet transforms are introduced. Briefly, we explain how transforms from these families can be used in conjunction with symmetric extension in order to handle signals of arbitrary length in a nonexpansive manner (which is often a desirable attribute in signal coding applications). The characteristics of the two transform families and their constituent transforms are then studied. For the more constrained of the two families, we identify precisely which transforms belong to the family (by specifying properties and conditions for membership). Such results might be exploited in the filter bank design process in order to find new symmetry-preserving reversible integer-to-integer wavelet transforms for signal coding applications.
\end{abstract}

\section{INTRODUCTION}

Lifting-based reversible integer-to-integer wavelet transforms [1, 2] have become a popular tool in signal coding applications. In such applications, however, it is often desirable to employ transforms that preserve symmetry. For example, symmetry-preserving transforms have the advantage of being compatible with symmetric extension techniques, allowing signals of arbitrary length to be handled in a nonexpansive manner [3]. (As a matter of terminology, a transform is said to be nonexpansive if its application to a signal of length $N$ always yields a result that can be completely characterized by no more than $N$ transform coefficients.) Fortunately, using the lifting scheme, one can construct transforms that are not only reversible and integer to integer, but also symmetry preserving (and nonexpansive).

In this paper, we introduce two families of symmetry-preserving reversible integer-to-integer wavelet transforms. Then, we proceed to study the characteristics of these families and their constituent transforms. In the case of the more constrained family, several interesting results are presented, which provide new insights into the transforms belonging to this family.

\section{NOTATION AND OTHER PRELIMINARIES}

Before proceeding further, a short digression concerning the notation used in this paper is appropriate. The symbols $\mathbb{Z}$ and $\mathbb{R}$ denote the sets of integer and real numbers, respectively. Matrix and vector quantities are indicated using bold type. The symbols $\mathbf{I}$ and $\mathbf{J}$ denote the identity and anti-identity matrices, respectively. In the case of matrix multiplication, we define the product operator as

$$
\prod_{i=M}^{N} \mathbf{A}_{i} \triangleq \mathbf{A}_{N} \mathbf{A}_{N-1} \cdots \mathbf{A}_{M+1} \mathbf{A}_{M}
$$

for $N \geq M$. (One should note the order in which the matrix factors are multiplied above, since matrix multiplication is not commutative.) In the case that $N<M$, we define the product operator notation to denote an "empty" product (i.e., the multiplicative identity, I).

This work was supported by the Natural Sciences and Engineering Research Council of Canada.
For a Laurent polynomial $P(z)$, we denote the degree of $P(z)$ as $\operatorname{deg} P(z)$, and define this quantity as follows. In the case that $P(z)$ has the form $P(z)=\sum_{i=M}^{N} p_{i} z^{-i}$ where $N \geq M, p_{M} \neq$ 0 , and $p_{N} \neq 0$ (i.e., $\left.P(z) \not \equiv 0\right), \operatorname{deg} P(z) \triangleq N-M$. In the case of the zero polynomial, we define $\operatorname{deg} 0 \triangleq-\infty$. Thus, for any two Laurent polynomials, $A(z)$ and $B(z)$, we have that $\operatorname{deg}(A(z) B(z))=\operatorname{deg} A(z)+\operatorname{deg} B(z)$.

For $\alpha \in \mathbb{R}$, the notation $\lfloor\alpha\rfloor$ denotes the largest integer not more than $\alpha$ (i.e., the floor function), and the notation $\lceil\alpha\rceil$ denotes the smallest integer not less than $\alpha$ (i.e., the ceiling function). The truncation, rounding-away-from-zero (RAFZ), and mod functions are defined, respectively, as

$$
\begin{gathered}
\operatorname{trunc} \alpha \triangleq\left\{\begin{array}{ll}
\lfloor\alpha\rfloor & \text { for } \alpha \geq 0 \\
\lceil\alpha\rceil & \text { for } \alpha<0
\end{array},\right. \\
\operatorname{rafz} \alpha \triangleq\left\{\begin{array}{ll}
\lceil\alpha\rceil & \text { for } \alpha \geq 0 \\
\lfloor\alpha\rfloor & \text { for } \alpha<0
\end{array},\right. \text { and }
\end{gathered}
$$

$\bmod (x, y) \triangleq x-y\lfloor x / y\rfloor, \quad$ where $\alpha \in \mathbb{R}$ and $x, y \in \mathbb{Z}$. A rounding operator $Q$ is said to be integer-shift invariant if

$$
Q(\alpha+x)=Q(\alpha)+x \quad \text { for all } \alpha \in \mathbb{R} \text { and all } x \in \mathbb{Z} .
$$

Similarly, a rounding operator $Q$ is said to be odd if

$$
Q(\alpha)=-Q(-\alpha) \text { for all } \alpha \in \mathbb{R} .
$$

One can show that a rounding operator cannot be both odd and integer-shift invariant [4]. In passing, we note that the floor and ceiling functions are integer-shift invariant (but not odd), while the trunc and rafz functions are odd (but not integer-shift invariant). All rounding operators considered in this paper are tacitly assumed to be memoryless, time invariant, and leave integer values unchanged. Any (reasonable) rounding operator will preserve signal symmetry (but not necessarily signal antisymmetry), while any odd rounding operator will preserve both signal symmetry and antisymmetry.

\section{TRANSFORM FAMILIES}

In this paper, we consider two families of symmetry-preserving reversible integer-to-integer wavelet transforms. Both are derived from the lifting-based parameterizations of linear-phase filter banks presented in [5], and have the general form shown in Fig. 1. In the figure, the $\left\{Q_{k}\right\}$ are rounding operators, and the analysis and synthesis polyphase filtering networks each consist of $2 \lambda$ lifting step filters $\left\{A_{k}\right\}$. As we will demonstrate, by choosing the lifting step filters $\left\{A_{k}\right\}$ wisely, one can construct filter banks that not only yield symmetric/antisymmetric subband signals (i.e., $y_{0}[n]$ and $y_{1}[n]$ ) for an appropriately chosen input signal (i.e., $x[n]$ ), but also yield intermediate signals (i.e., $\left\{u_{k}[n]\right\},\left\{v_{k}[n]\right\}$ ) that are all or mostly symmetric/antisymmetric.

The first family of reversible integer-to-integer wavelet transforms is associated with a linear-phase filter bank having oddlength analysis/synthesis filters, and has been described in [5]. For convenience, we will refer to this as the odd-length analy- 


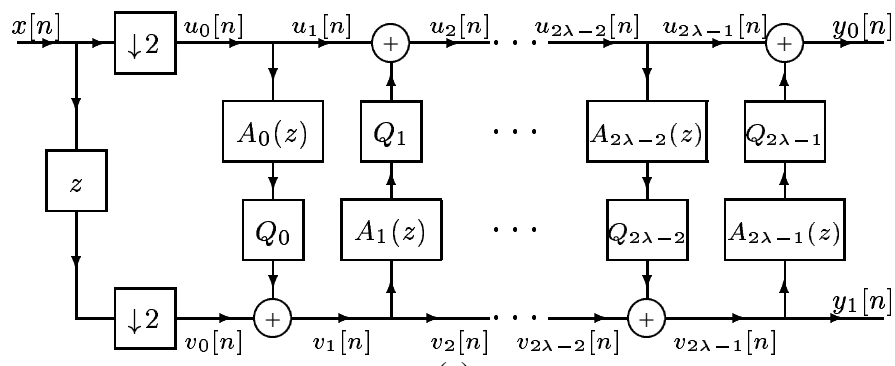

(a)

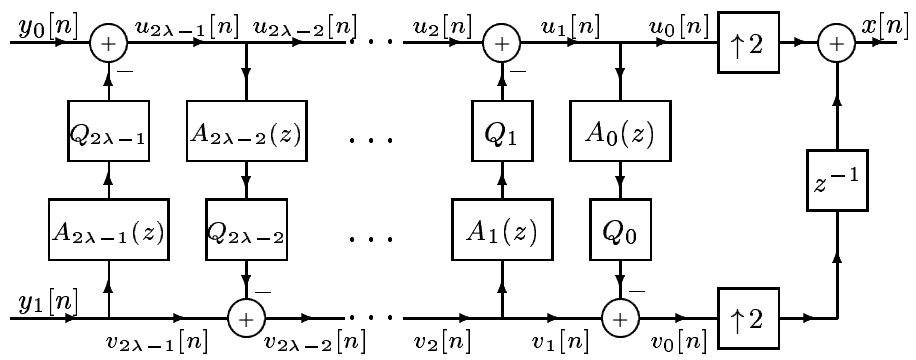

(b)

Fig. 1. Lifting-based realization of reversible integer-to-integer wavelet transform. (a) Analysis filter bank and (b) synthesis filter bank.

sis/synthesis filter (OLASF) parameterization. In this case, the lifting step filters are chosen to have transfer functions of the form:

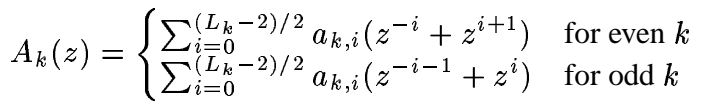

for $k=0,1, \ldots, 2 \lambda-1$, where the $\left\{L_{k}\right\}$ are all even integers. Without loss of generality, we assume that none of the $\left\{A_{k}(z)\right\}_{k=1}^{2 \lambda-2}$ are identically zero. That is, only $A_{0}(z)$ and $A_{2 \lambda-1}(z)$ may be identically zero. The $\left\{Q_{k}\right\}_{k=0}^{2 \lambda-1}$ are chosen to be any arbitrary rounding operators (e.g., the floor, ceiling, rafz, or trunc functions).

The second family of reversible integer-to-integer wavelet transforms is associated with a linear-phase filter bank having evenlength analysis/synthesis filters, and has been described in [5]. For convenience, we will refer to this as the even-length analysis/synthesis filter (ELASF) parameterization. In this case, the lifting step filters are chosen to have transfer functions of the form:

$$
A_{k}(z)= \begin{cases}-1 & \text { for } k=0 \\ \frac{1}{2}+\hat{A}_{1}(z) & \text { for } k=1 \\ \hat{A}_{k}(z) & \text { for } k \geq 2\end{cases}
$$

for $k=0,1, \ldots, 2 \lambda-1$, where

$$
\hat{A}_{k}(z)=\sum_{i=1}^{\left(L_{k}-1\right) / 2} \hat{a}_{k, i}\left(z^{-i}-z^{i}\right),
$$

$\lambda \geq 1$, and the $\left\{L_{k}\right\}$ are all odd integers. Without loss of generality, we assume that none of the $\left\{\hat{A}_{k}(z)\right\}_{k=2}^{2 \lambda-2}$ are identically zero. That is, only $\hat{A}_{1}(z)$ and $\hat{A}_{2 \lambda-1}(z)$ may be identically zero. The operator $Q_{0}$ is simply the identity (since the output of the filter $A_{0}$ is always an integer), and the rounding operator $Q_{1}$ is chosen to be integer-shift invariant (e.g., the floor or ceiling function). The remaining rounding operators $\left\{Q_{k}\right\}_{k=2}^{2 \lambda-1}$ are chosen to be odd (e.g., the trunc or rafz function) for even $\bar{k}$ and chosen arbitrarily for odd $k$. (We note that, in the case of transform families with a similar structure, it is commonly suggested that the $\left\{Q_{k}\right\}$ must be chosen as odd functions for all $k>2$ (e.g., as in [6]). Such a choice, however, is overly restrictive.)

\section{TRANSFORMS AND SYMMETRIC EXTENSION}

In the previous section, we introduced the OLASF and ELASF families of reversible integer-to-integer wavelet transforms. Now, we briefly explain how transforms from these two families can be used with symmetric extension in order to handle signals of arbitrary length in a nonexpansive manner.

Consider a filter bank of the form shown in Fig. 1 that is constrained to be of the OLASF type. Suppose that we are given a signal $\hat{x}[n]$ defined for $n=0,1, \ldots, N-1$. We then choose $x[n]$, the input to the analysis filter bank, as the following infinite-length signal which is a symmetric extension of $\hat{x}[n]$ :

$x[n]=\hat{x}[\min (\bmod (n, 2 N-2), 2 N-2-\bmod (n, 2 N-2))]$

(i.e., $x[n]$ is defined such that $x[n]=x[n+2 N-2]$ and $x[n]=$ $x[-n])$. With this choice of extension, one can show (e.g., as in [7]) that the $\left\{u_{k}[n]\right\}_{k=0}^{2 \lambda-1}$ and $y_{0}[n]$ are all $(N-1)$-periodic and symmetric about 0 and $\frac{N-1}{2}$. Likewise, the $\left\{v_{k}[n]\right\}_{k=0}^{2 \lambda-1}$ and $y_{1}[n]$ are all $(N-1)$-periodic and symmetric about $-\frac{1}{2}$ and $\frac{N-2}{2}$. As a result, the $\left\{u_{k}[n]\right\}_{k=0}^{2 \lambda-1}$ and $y_{0}[n]$ are completely characterized by their samples at indices $0,1, \ldots,\left\lfloor\frac{N-1}{2}\right\rfloor$, and the $\left\{v_{k}[n]\right\}_{k=0}^{2 \lambda-1}$ and $y_{1}[n]$ are completely characterized by their samples at indices $0,1, \ldots,\left\lfloor\frac{N-2}{2}\right\rfloor$. Consequently, for even $N$, we require $\frac{N}{2}$ samples to represent each of $y_{0}[n]$ and $y_{1}[n]$, and for odd $N$, we need $\frac{N+1}{2}$ samples to represent $y_{0}[n]$ and $\frac{N-1}{2}$ samples to represent $y_{1}[n]$. In either case, a total of $N$ samples is required, and the resulting transform is, therefore, nonexpansive.

Consider a filter bank of the form shown in Fig. 1 that is constrained to be of the ELASF type. Suppose that we are given a signal $\hat{x}[n]$ defined for $n=0,1, \ldots, N-1$. We then choose $x[n]$, the input to the analysis filter bank, as the following infinite-length signal which is a symmetric extension of $\hat{x}[n]$ :

$$
x[n]=\hat{x}[\min (\bmod (n, 2 N), 2 N-1-\bmod (n, 2 N))]
$$

(i.e., $x[n]$ is defined such that $x[n]=x[n+2 N]$ and $x[n]=$ $x[-1-n]$ ). With this choice of extension, one can show (e.g., as in [7]) that the $\left\{u_{k}[n]\right\}_{k=2}^{2 \lambda-1}$ and $y_{0}[n]$ are $N$-periodic and symmetric with symmetry centers $-\frac{1}{2}$ and $\frac{N-1}{2}$, and the $\left\{v_{k}[n]\right\}_{i=1}^{2 \lambda-1}$ and $y_{1}[n]$ are $N$-periodic and antisymmetric with the same symmetry centers. As a result, the $\left\{u_{k}[n]\right\}_{k=2}^{2 \lambda-1}$ and $y_{0}[n]$ are completely characterized by their samples at indices $0,1, \ldots,\left\lfloor\frac{N-1}{2}\right\rfloor$ and the $\left\{v_{k}[n]\right\}_{k=1}^{2 \lambda-1}$ and $y_{1}[n]$ are completely characterized by their samples at indices $0,1, \ldots,\left\lfloor\frac{N-2}{2}\right\rfloor$. Consequently, for even $N$, we require $\frac{N}{2}$ samples to represent each of $y_{0}[n]$ and $y_{1}[n]$, and for odd $N$, we require $\frac{N+1}{2}$ samples to represent $y_{0}[n]$ and $\frac{N-1}{2}$ samples to represent $y_{1}[n]$. In either case, regardless of the parity of $N$, a total of $N$ samples is required, and the resulting transform is, therefore, nonexpansive.

\section{OLASF FAMILY}

Any perfect-reconstruction (PR) linear-phase FIR (2-channel) filter bank with odd-length analysis/synthesis filters has a corresponding OLASF parameterization, provided that the analysis and synthesis filters are normalized appropriately (via a scaling and shift of their impulse responses). (For example, the lowpass and highpass analysis filters must have impulse responses centered about 0 and -1 , respectively.) The proof is constructive by a matrix Euclidean algorithm (e.g., by using an algorithm based on that described in [8]). 


\section{ELASF FAMILY}

Only a subset of all PR linear-phase FIR (2-channel) filter banks with even-length analysis/synthesis filters have corresponding ELASF parameterizations. Moreover, this subset is quite small, as there are many such filter banks that cannot be realized in this way. In what follows, we examine some of the characteristics of transforms in the ELASF family, and in so doing, demonstrate some of the reasons for the incompleteness of this parameterization.

\subsection{Transform Properties}

Consider the linear version of a filter bank of the form shown in Fig. 1(a) that is constrained to be of the ELASF type as defined by (2). (By "linear version", we mean the filter bank obtained by simply setting all of the $\left\{Q_{k}\right\}$ equal to the identity.) Let us denote the corresponding lowpass and highpass analysis filter transfer functions as $H_{0}(z)$ and $H_{1}(z)$, respectively. Further, we define the transfer matrix for part of the polyphase filtering network as follows:

$$
\begin{aligned}
\mathbf{P}(z) & \triangleq\left[\begin{array}{ll}
P_{0,0}(z) & P_{0,1}(z) \\
P_{1,0}(z) & P_{1,1}(z)
\end{array}\right] \\
& =\left(\prod_{k=1}^{\lambda-1}\left[\begin{array}{cc}
1 & \hat{A}_{2 k+1}(z) \\
0 & 1
\end{array}\right]\left[\begin{array}{cc}
1 & 0 \\
\hat{A}_{2 k}(z) & 1
\end{array}\right]\right)\left[\begin{array}{cc}
1 & \hat{A}_{1}(z) \\
0 & 1
\end{array}\right]
\end{aligned}
$$

Using noble identities and straightforward algebraic manipulation, we can show

$$
\left[\begin{array}{l}
H_{0}(z) \\
H_{1}(z)
\end{array}\right]=\mathbf{P}\left(z^{2}\right)\left[\begin{array}{ll}
1 & \frac{1}{2} \\
0 & 1
\end{array}\right]\left[\begin{array}{cc}
1 & 0 \\
-1 & 1
\end{array}\right]\left[\begin{array}{l}
1 \\
z
\end{array}\right]=\mathbf{P}\left(z^{2}\right)\left[\begin{array}{c}
\frac{1}{2}(z+1) \\
z-1
\end{array}\right]
$$

For convenience, can rewrite the preceding equation as follows:

$$
\begin{aligned}
& H_{0}(z)=\frac{1}{2}(z+1) P_{0,0}\left(z^{2}\right)+(z-1) P_{0,1}\left(z^{2}\right) \\
& H_{1}(z)=(z-1) P_{1,1}\left(z^{2}\right)+\frac{1}{2}(z+1) P_{1,0}\left(z^{2}\right)
\end{aligned}
$$

A detailed analysis of (3) reveals that $\mathbf{P}(z)$ has the following important properties:

1. $P_{0,0}(z)$ and $P_{1,1}(z)$ both have coefficient sequences that are symmetric about 0 ;

2. $P_{0,1}(z)$ and $P_{1,0}(z)$ both have coefficient sequences that are antisymmetric about 0 ;

3. For $i=0,1$ and $j=0,1, \operatorname{deg} P_{i, j}(z)$ is even, except when $P_{i, j}(z) \equiv 0 ; \operatorname{deg} P_{0,0}(z) \geq 0$ and $\operatorname{deg} P_{1,1}(z) \geq 0$.

4. If at least one of the $\left\{\hat{A}_{k}(z)\right\}$ is not identically zero, we have that either: $\operatorname{deg} P_{0,0}(z)>\operatorname{deg} P_{0,1}(z)$ and $\operatorname{deg} P_{1,0}(z)>$ $\operatorname{deg} P_{1,1}(z)$; or $\operatorname{deg} P_{0,0}(z)<\operatorname{deg} P_{0,1}(z)$ and $\operatorname{deg} P_{1,0}(z)<$ $\operatorname{deg} P_{1,1}(z)$;

5. $\operatorname{deg} P_{0,0}(z) \neq \operatorname{deg} P_{0,1}(z), \operatorname{deg} P_{1,0}(z) \neq \operatorname{deg} P_{1,1}(z)$, $\operatorname{deg} P_{0,0}(z) \neq \operatorname{deg} P_{1,0}(z)$, and $\operatorname{deg} P_{0,1}(z) \neq \operatorname{deg} P_{1,1}(z)$;

6. $P_{0,0}(1)=1$ and $P_{1,1}(1)=1$.

The above properties will be used extensively in what follows. To begin, however, we will examine the form of $H_{0}(z)$ and $H_{1}(z)$, in light of the above properties.

For convenience, let us denote the first and second terms in the expression for $H_{0}(z)$ in (4a) as $B(z)$ and $C(z)$, respectively. That is, we define $B(z) \triangleq \frac{1}{2}(z+1) P_{0,0}\left(z^{2}\right)$ and $C(z) \triangleq(z-$ 1) $P_{0,1}\left(z^{2}\right)$. Due to the form of $P_{0,0}\left(z^{2}\right)$ (which is implied by property 1 of $\mathbf{P}(z)), B(z)$ has a coefficient sequence $b[n]$ that is symmetric about $-\frac{1}{2}$ with adjacent pairs of samples being equal in value (i.e., $b[2 n]=b[2 n-1]$ ). Likewise, due to the form of $P_{0,1}\left(z^{2}\right)$ (which is implied by property 2 of $\mathbf{P}(z)$ ), $C(z)$ has a coefficient sequence $c[n]$ that is symmetric about $-\frac{1}{2}$ with adjacent pairs of samples being equal in magnitude but opposite in sign (i.e., $c[2 n]=-c[2 n-1]$ ). Suppose that $P_{0,1}(z) \not \equiv 0$. In this case, from properties 3 and 5 of $\mathbf{P}(z)$, we know that $\operatorname{deg} P_{0,0}\left(z^{2}\right)$ and $\operatorname{deg} P_{0,1}\left(z^{2}\right)$ must differ by a nonzero integer multiple of 4 . Since $H_{0}(z)=B(z)+C(z), H_{0}(z)$ must have a coefficient sequence that is symmetric about $-\frac{1}{2}$ and begins and ends with pairs of coefficients that are either equal or equal in magnitude but opposite in sign. In the degenerate case, in which $P_{0,1}(z) \equiv 0$, we simply have $H_{0}(z)=\frac{1}{2}(z+1)$. By considering the degrees of $B(z)$ and $C(z)$, we can also see that

$$
\operatorname{deg} H_{0}(z)=1+2 \max \left(\operatorname{deg} P_{0,0}(z), \operatorname{deg} P_{0,1}(z)\right)
$$

Since, by property 3 of $\mathbf{P}(z), \operatorname{deg} P_{0,0}(z)$ is always even and $\operatorname{deg} P_{0,1}(z)$ is even (except when $P_{0,1}(z) \equiv 0$ ), we have that $\operatorname{deg} H_{0}(z)$ is odd. Thus, as had been suggested earlier, $H_{0}$ is an even-length filter.

For convenience, let us denote the first and second terms in the expression for $H_{1}(z)$ in $(4 \mathrm{~b})$ as $B(z)$ and $C(z)$, respectively. That is, we define $B(z) \triangleq(z-1) P_{1,1}\left(z^{2}\right)$ and $C(z) \triangleq \frac{1}{2}(z+$ 1) $P_{1,0}\left(z^{2}\right)$. Due to the form of $P_{1,1}\left(z^{2}\right)$ (which is implied by property 1 of $\mathbf{P}(z)), B(z)$ has a coefficient sequence $b[n]$ that is antisymmetric about $-\frac{1}{2}$ with adjacent pairs of samples being equal in magnitude but opposite in sign (i.e., $b[2 n]=-b[2 n-1]$ ). Likewise, due to the form of $P_{1,0}\left(z^{2}\right)$ (which is implied by property 2 of $\mathbf{P}(z)), C(z)$ has a coefficient sequence $c[n]$ that is antisymmetric about $-\frac{1}{2}$ with adjacent pairs of samples being equal (i.e., $c[2 n]=c[2 n-1])$. Suppose that $P_{1,0}(z) \not \equiv 0$. In this case, from properties 3 and 5 of $\mathbf{P}(z)$, we know that $\operatorname{deg} P_{1,1}\left(z^{2}\right)$ and $\operatorname{deg} P_{1,0}\left(z^{2}\right)$ must differ by a nonzero integer multiple of 4 . Since $H_{1}(z)=B(z)+C(z), H_{1}(z)$ must have a coefficient sequence that is antisymmetric about $-\frac{1}{2}$ and begins and ends with pairs of coefficients that are either equal or equal in magnitude but opposite in sign. In the degenerate case, in which $P_{1,0}(z) \equiv 0$, we simply have $H_{1}(z)=z-1$. By examining the degrees of $B(z)$ and $C(z)$, we can see that

$$
\operatorname{deg} H_{1}(z)=1+2 \max \left(\operatorname{deg} P_{1,1}(z), \operatorname{deg} P_{1,0}(z)\right)
$$

Since, by property 3 of $\mathbf{P}(z), \operatorname{deg} P_{1,1}(z)$ is always even and $\operatorname{deg} P_{1,0}(z)$ is even (except when $P_{1,0}(z) \equiv 0$ ), we have that $\operatorname{deg} H_{1}(z)$ is odd. Thus, as had been suggested earlier, $H_{1}$ is an even-length filter.

The above results are significant as they show that $H_{0}(z)$ and $H_{1}(z)$ both have a highly structured form (i.e., their coefficient sequences are each the sum of two highly structured sequences). Examining the expression for $\operatorname{deg} H_{0}(z)$ and $\operatorname{deg} H_{1}(z)$, we can make one further observation regarding the analysis filters. That is, except in the degenerate case in which all of the $\left\{\hat{A}_{k}(z)\right\}$ are identically zero, the analysis filters cannot have the same length. To see why this is so, we proceed as below.

Since, by assumption, at least one of the $\left\{\hat{A}_{k}(z)\right\}$ is not identically zero, property 4 of $\mathbf{P}(z)$ implies that two cases are possible: 1) $\operatorname{deg} P_{0,0}(z)>\operatorname{deg} P_{0,1}(z)$ and $\operatorname{deg} P_{1,0}(z)>\operatorname{deg} P_{1,1}(z)$; or 2) $\operatorname{deg} P_{0,0}(z)<\operatorname{deg} P_{0,1}(z)$ and $\operatorname{deg} P_{1,0}(z)<\operatorname{deg} P_{1,1}(z)$. In the first case, we have from (5) and (6) that $\operatorname{deg} H_{0}(z)=$ $1+2 \operatorname{deg} P_{0,0}(z)$ and $\operatorname{deg} H_{1}(z)=1+2 \operatorname{deg} P_{1,0}(z)$. From property 5 of $P(z)$, however, we know that $\operatorname{deg} P_{0,0}(z) \neq \operatorname{deg} P_{1,0}(z)$. Therefore, $\operatorname{deg} H_{0}(z) \neq \operatorname{deg} H_{1}(z)$. In the second case, we have from (5) and (6) that $\operatorname{deg} H_{0}(z)=1+2 \operatorname{deg} P_{0,1}(z)$ and $\operatorname{deg} H_{1}(z)=$ $1+2 \operatorname{deg} P_{1,1}(z)$. From property 5 of $\mathbf{P}(z)$, however, we know that $\operatorname{deg} P_{0,1}(z) \neq \operatorname{deg} P_{1,1}(z)$. Therefore, $\operatorname{deg} H_{0}(z) \neq \operatorname{deg} H_{1}(z)$. By combining the results for the above two cases, we have that $\operatorname{deg} H_{0}(z) \neq \operatorname{deg} H_{1}(z)$, except in the degenerate case in which all of the $\left\{\hat{A}_{k}(z)\right\}$ are identically zero. Consequently, the analysis filters cannot have the same lengths, except in this degenerate case.

In order to belong to the ELASF family, a transform must have analysis filters of the form described above. Obviously, the close relationship between pairs of samples in the analysis filter impulse responses is quite constraining. For this reason, the ELASF family 
cannot be a complete parameterization of all PR linear-phase FIR (2-channel) filter banks with even-length filters. Furthermore, an even more basic reason exists for this lack of completeness. As noted above, the analysis filters cannot be of equal length, except in the degenerate case in which all of the $\left\{\hat{A}_{k}(z)\right\}$ are identically zero.

Consider now the DC and Nyquist gains of the analysis filters, $H_{0}$ and $H_{1}$. From property 2 of $\mathbf{P}(z)$, we know that $P_{0,1}(z)$ and $P_{1,0}(z)$ both have antisymmetric coefficient sequences, and consequently,

$$
\left.P_{0,1}\left(z^{2}\right)\right|_{z= \pm 1}=0 \quad \text { and }\left.\quad P_{1,0}\left(z^{2}\right)\right|_{z= \pm 1}=0 .
$$

From property 6 of $\mathbf{P}(z)$, we have that $P_{0,0}(1)=1$ and $P_{1,1}(1)=$ 1 , and consequently,

$$
\left.P_{0,0}\left(z^{2}\right)\right|_{z= \pm 1}=1 \quad \text { and }\left.\quad P_{1,1}\left(z^{2}\right)\right|_{z= \pm 1}=1 .
$$

Using (7) and (8), we can deduce from (4) that

$$
\begin{gathered}
H_{0}(1)=1, \quad H_{0}(-1)=0, \\
H_{1}(-1)=-2, \quad H_{1}(1)=0 .
\end{gathered}
$$

Thus, for any transform in the ELASF family, the associated lowpass analysis filter must have DC and Nyquist gains of 1 and 0 , respectively, while the associated highpass analysis filter must have Nyquist and DC gains of 2 and 0 , respectively. This result is of practical interest, since it is often desirable for a reversible integerto-integer wavelet transform to have a corresponding lowpass analysis filter with a DC gain of 1 (which typically results in a transform with good dynamic range properties).

\subsection{Swapping Analysis and Synthesis Filters}

Assume that we have a filter bank from the ELASF family. Suppose now that we swap the analysis and synthesis filters, allowing the filters to be renormalized in the process. This renormalization can always be performed in such a way that the resulting filter bank also belongs to the ELASF family. (To date, this fact seems to have been overlooked (e.g., as in [6]).) In what follows, we now prove our above assertion regarding the exchange of analysis and synthesis filters.

Consider the linear version of a filter bank of the form shown in Fig. 1 that is constrained to be of the ELASF type as defined by (2). Let us denote the analysis filter transfer functions as $H_{0}(z)$ and $H_{1}(z)$ and the corresponding synthesis filter transfer functions as $F_{0}(z)$ and $F_{1}(z)$. From the diagram, we can see that the analysis polyphase matrix, $\mathbf{E}(z)$, is given by

$$
\begin{gathered}
\mathbf{E}(z)=\left(\prod_{k=1}^{\lambda-1}\left[\begin{array}{cc}
1 & \hat{A}_{2 k+1}(z) \\
0 & 1
\end{array}\right]\left[\begin{array}{cc}
1 & 0 \\
\hat{A}_{2 k}(z) & 1
\end{array}\right]\right)\left[\begin{array}{cc}
1 & \hat{A}_{1}(z) \\
0 & 1
\end{array}\right] \\
{\left[\begin{array}{ll}
1 & \frac{1}{2} \\
0 & 1
\end{array}\right]\left[\begin{array}{cc}
1 & 0 \\
-1 & 1
\end{array}\right]}
\end{gathered}
$$

Suppose that we now construct a new filter bank with the analysis filters $H_{0}^{\prime}(z)$ and $H_{1}^{\prime}(z)$ where $H_{0}^{\prime}(z)=\alpha_{0} z F_{0}(z)$ and $H_{1}^{\prime}(z)=$ $\alpha_{1} z F_{1}(z)$. In other words, the new analysis filters are chosen to be renormalized versions of the synthesis filters from the original filter bank. Further assume that we continue to employ the same polyphase representation for the new filter bank. Let us denote the new analysis polyphase matrix as $\mathbf{E}^{\prime}(z)$. From the definition of the polyphase representation, we can show

$$
\mathbf{E}^{\prime}(z)=\left[\begin{array}{cc}
\alpha_{0} & 0 \\
0 & \alpha_{1}
\end{array}\right]\left(\mathbf{E}^{-1}(z)\right)^{T} \mathbf{J}
$$

Substituting (9) in (10), we obtain

$$
\begin{gathered}
\mathbf{E}^{\prime}(z)=\left[\begin{array}{cc}
\alpha_{0} & 0 \\
0 & \alpha_{1}
\end{array}\right]\left(\prod_{k=0}^{\lambda-1}\left[\begin{array}{cc}
1 & 0 \\
-\hat{A}_{2 k+1}(z) & 1
\end{array}\right]\left[\begin{array}{cc}
1 & -\hat{A}_{2 k}(z) \\
0 & 1
\end{array}\right]\right) \\
{\left[\begin{array}{cc}
1 & 0 \\
-\hat{A}_{1}(z) & 1
\end{array}\right]\left[\begin{array}{cc}
1 & 0 \\
-\frac{1}{2} & 1
\end{array}\right]\left[\begin{array}{ll}
1 & 1 \\
0 & 1
\end{array}\right] \mathbf{J}}
\end{gathered}
$$

Suppose now that we choose $\alpha_{0}=\frac{1}{2}$ and $\alpha_{1}=-2$. In this case, we can rewrite (11) as follows:

$$
\begin{aligned}
\mathbf{E}^{\prime}(z)= & {\left[\begin{array}{cc}
\frac{1}{2} & 0 \\
0 & -2
\end{array}\right]\left(\prod_{k=1}^{\lambda-1}\left[\begin{array}{cc}
1 & 0 \\
-\hat{A}_{2 k+1}(z) & 1
\end{array}\right]\left[\begin{array}{cc}
1 & -\hat{A}_{2 k}(z) \\
0 & 1
\end{array}\right]\right) } \\
& {\left[\begin{array}{cc}
1 & 0 \\
-\hat{A}_{1}(z) & 1
\end{array}\right]\left[\begin{array}{cc}
1 & 0 \\
-\frac{1}{2} & 1
\end{array}\right]\left[\begin{array}{ll}
1 & 1 \\
0 & 1
\end{array}\right] \mathbf{J} } \\
= & {\left[\begin{array}{cc}
\frac{1}{2} & 0 \\
0 & -2
\end{array}\right]\left(\prod_{k=1}^{\lambda-1}\left[\begin{array}{cc}
1 & 0 \\
-\hat{A}_{2 k+1}(z) & 1
\end{array}\right]\left[\begin{array}{cc}
1 & -\hat{A}_{2 k}(z) \\
0 & 1
\end{array}\right]\right) } \\
& \quad\left[\begin{array}{cc}
1 & 0 \\
-\hat{A}_{1}(z) & 1
\end{array}\right]\left[\begin{array}{cc}
2 & 0 \\
0 & -\frac{1}{2}
\end{array}\right]\left[\begin{array}{ll}
1 & \frac{1}{2} \\
0 & 1
\end{array}\right]\left[\begin{array}{cc}
1 & 0 \\
-1 & 1
\end{array}\right] \\
= & \left(\prod_{k=1}^{\lambda-1}\left[\begin{array}{cc}
1 & 0 \\
4 \hat{A}_{2 k+1}(z) & 1
\end{array}\right]\left[\begin{array}{cc}
1 & \frac{1}{4} \hat{A}_{2 k}(z) \\
0 & 1
\end{array}\right]\right)\left[\begin{array}{cc}
1 & 0 \\
4 \hat{A}_{1}(z) & 1
\end{array}\right] \\
& {\left[\begin{array}{ll}
1 & \frac{1}{2} \\
0 & 1
\end{array}\right]\left[\begin{array}{cc}
1 & 0 \\
-1 & 1
\end{array}\right] }
\end{aligned}
$$

Thus, the new analysis polyphase matrix, $\mathbf{E}^{\prime}(z)$, has the same general form as the original one. In other words, the new filter bank also has a symmetry-preserving reversible integer-to-integer implementation (which belongs to the ELASF family). The above result is practically useful, since, in some cases, the "transposed" filter bank (i.e., the one with the analysis and synthesis filters swapped) may also be effective for coding purposes.

\section{CONCLUSIONS}

Two families of symmetry-preserving reversible integer-to-integer wavelet transforms were introduced (i.e., the OLASF and ELASF families), and we explained how transforms from these families can be used in conjunction with symmetric extension in order to handle signals of arbitrary length in a nonexpansive manner. The characteristics of the two transform families and their constituent transforms were then studied. For the more constrained of the two families, we characterized the transforms belonging to this family. That is, we showed that: 1) such transforms are associated with analysis filters having transfer functions of a highly structured form; 2) the DC and Nyquist gains of the analysis filters are fixed, independent of the choice of lifting step filters; and 3) if a particular filter bank is associated with a transform in the ELASF family, then so too is its "transposed" version. By better understanding the characteristics of this family of transforms, one can hope to better utilize this family in signal coding applications.

\section{REFERENCES}

[1] A. R. Calderbank, I. Daubechies, W. Sweldens, and B.-L. Yeo, "Wavelet transforms that map integers to integers," Applied and Computational Harmonic Analysis, vol. 5, no. 3, pp. 332-369, July 1998.

[2] ISO/IEC, ISO/IEC 15444-1: Information technology-JPEG 2000 image coding system-Part 1: Core coding system, 2001.

[3] C. M. Brislawn, 'Preservation of subband symmetry in multirate signal coding," IEEE Trans. on Signal Processing, vol. 43, no. 12 , pp. 3046-3050, Dec. 1995.

[4] M. D. Adams and F. Kossentini, "Generalized S transform," in Proc. of IEEE ICASSP, 2001, pp. 1749-1752.

[5] M. D. Adams and F. Kossentini, 'Low-complexity reversible integer-to-integer wavelet transforms for image coding," in Proc. of IEEE Pacific Rim Conference, Victoria, BC, Canada, Aug. 1999, pp. 177-180.

[6] C. Brislawn and B. Wohlberg, 'Boundary extensions and reversible implementation for half-sample symmetric filter banks," ISO/IEC JTC 1/SC 29/WG 1 N 2119, Mar. 2001.

[7] M. D. Adams and R. Ward, 'Symmetry-preserving reversible integer-to-integer wavelet transforms," Submitted to IEEE ICASSP 2002, 2001.

[8] I. Daubechies and W. Sweldens, 'Factoring wavelet transforms into lifting steps," Journal of Fourier Analysis and Applications, vol. 4, pp. 247-269, 1998 